\title{
Arp 299: The show must go on!
}

\section{Miguel Pérez-Torres* ${ }^{\dagger}$}

IAA-CSIC, Glorieta de la Astronomía s/n, 18008 Granada, Spain

E-mail: torres@iaa.es

Luminous and Ultra-Luminous Infrared Galaxies (U/LIRGs) are also strong emitters of both thermal (free-free) and non-thermal (mainly synchrotron) radio emission. This is very handy since, unlike optical and infra-red observations, radio is not obscured by the ubiquitous dust present in U/LIRGs, which allows a direct view of the ongoing activity inside those prolific star-forming galaxies.

In this talk, I present some selected results on what is probably the most spectacular supernova factory in a nearby LIRG, Arp 299. Namely, I show (i) the impressive discovery of an extremely prolific supernova factory in the central $\sim 150$ pc of the galaxy Arp 299-A ( $D=45 \mathrm{Mpc}$ ), showing that essentially all core-collapse supernovae explode in the central $150 \mathrm{pc}$ of the galaxy, (ii) the detection and precise location of the long-sought AGN in Arp 299-A, and (iii) the monitoring of the compact sources in Arp 299-A. A movie summarizing those findings can be found in the following URL: http://www.iaa.es/ torres/research/arp299a.html. All those results demonstrate that very-high angular resolution studies of nearby U/LIRGs are of high relevance for the comprehension of both local and high-z starbursting galaxies, and that Arp 299 stands itself as a fundamental piece of such studies.

11th European VLBI Network Symposium \& Users Meeting,

October 9-12, 2012

Bordeaux, France

\footnotetext{
* Speaker.

${ }^{\dagger}$ Based on results obtained with the European VLBI Network (EVN).
} 


\section{Introduction}

A large fraction of the massive star-formation at both low- and high- $z$ has taken place in Luminous and Ultra-luminous Infrared Galaxies (U/LIRGs) [7], as their star-formation rates (SFR) are formidable in comparison with those in field galaxies. Indeed, by applying evolutionary stellar models to bursts of age 10-100 Myr, and adopting a standard initial mass function (IMF), one can obtain the SFR as a function of its far-infrared luminosity $\left(L_{\mathrm{FIR}}\right)[6]$ :

$$
\mathrm{SFR} \approx 17.2\left(\frac{\mathrm{L}_{\mathrm{FIR}}}{10^{11} \mathrm{~L} \odot}\right) \mathrm{M}_{\odot} \mathrm{yr}^{-1}
$$

While the compact, centrally located radio emission in U/LIRGs might be generated by a point-like source, i.e. an active galactic nucleus (AGN), or by the combined effect of multiple radio supernovae ( $\mathrm{RSNe}$ ) and/or supernova remnants ( $\mathrm{SNRs}$ ), it is well established that in the circumnuclear regions of U/LIRGs there is an ongoing burst of (massive) star formation. Since stars more massive than $\mathrm{M} \gtrsim 8 \mathrm{M}_{\odot}$ explode as core-collapse supernovae (CCSNe), the observed rate at which those stars die $\left(r_{\mathrm{CCSN}}\right)$ can be used as a direct measurement of the SFR in galaxies, and may provide unique information about the IMF of the high-mass stars. In fact, if for the sake of simplicity we assume a constant star-formation rate for a starburst, it can be shown that (e.g. [11])

$$
r_{\mathrm{CCSN}}=\operatorname{SFR}\left(\frac{\alpha-2}{\alpha-1}\right)\left(\frac{m_{\mathrm{SN}}^{1-\alpha}-m_{u}^{1-\alpha}}{m_{l}^{2-\alpha}-m_{u}^{2-\alpha}}\right)
$$

where $S F R$ is the constant star formation rate in $\mathrm{M}_{\odot} \mathrm{yr}^{-1}, \alpha$ is the power-law coefficient of the initial mass function (which states that the number of stars with mass $m$ is proportional to $m^{-\alpha}$ ), $m_{l}$ and $m_{u}$ are the lower and upper mass limits of the stars, and $m_{\mathrm{SN}}$ is the minimum mass of stars that yield supernovae.

However, the direct detection of CCSNe in the extreme densities of the central few hundred pc of U/LIRGs is, as pointed out earlier, extremely difficult since emission in the visual band suffers very significant extinction in those regions, which contain large amounts of dust, and can at best yield only a lower limit to the true value of $r_{\mathrm{CCSN}}$. Fortunately, radio is not affected by dust absorption. Moreover, significant radio emission from CCSNe is expected-as opposed to thermonuclear SNe-as the interaction of the SN ejecta with the circumstellar medium (CSM) gives rise to a high-energy density shell, which is Rayleigh-Taylor unstable and drives turbulent motions that amplify the existing magnetic field in the presupernova wind, and efficiently accelerate relativistic electrons, thus enhancing the emission of synchrotron radiation at radio wavelengths [3].

Thus, starburst activity in the circumnuclear regions of LIRGs ensures both the presence of a high number of massive stars and a dense surrounding medium, so bright radio $\mathrm{SNe}$ are expected to occur. Those SNe can be detected, even in the innermost regions of U/LIRGs, by means of high angular resolution $(\leq 0.05$ arcsec), high-sensitivity $(\leq 0.05 \mathrm{mJy})$ radio searches, which proved that they can unveil nuclear starbursts and detect individual radio supernovae up to large distances in the local Universe (e.g. Arp 220 [9]; Arp 299 [10, 2]; IC 883 [14]). 


\section{Arp 299 rocks!}

The merging system Arp $299(\mathrm{D}=44.8 \mathrm{Mpc}$ ) is the most luminous infrared galaxy in the nearby $50 \mathrm{Mpc}$, with $\log \left(\mathrm{L}_{\mathrm{FIR}} / \mathrm{L}_{\odot}\right)>11.5$. An active starburst in Arp 299 is indicated by the high frequency of recent optically discovered supernovae in this galaxy, including SN 1992bu, SN 1993G, SN 1998T, SN 1999D, SN 2005U, SN 2010O, and SN 2010P (see e.g. [13] and references therein). Based on its infrared luminosity $\left(L_{I R} \approx 6.5 \times 10^{11} L_{\odot}\right)$, the implied CCSN rate for the whole Arp 299 system is $\approx 1.8 \mathrm{SN} / \mathrm{yr}$. Since about $50 \%$ of its total infrared emission comes from Arp 299-A, this makes of it an obvious target for searching recently exploded CCSNe.

Optical and near-infrared observations are likely to miss a significant fraction of CCSNe in the innermost regions of Arp 299-A due to high values of extinction $\left(A_{V} \sim 34-40\right)$ and the lack of the necessary angular resolution. Therefore, radio observations of Arp 299-A at high angular resolution, high sensitivity are the only promising way of detecting new CCSNe, and thus measuring directly its CCSN rate and SFR. Very Long Baseline Array (VLBA) observations carried out during 2002 and 2003 resulted in the detection of five compact sources [8], one of which (A0) was identified as a young $\mathrm{SN}$.

\subsection{An extremely prolific supernova factory in Arp 299-A unveiled with the EVN}

In 2008, we used the eEVN at 5.0 GHz to image the central region of Arp 299-A at high resolution and with high sensitivity. These observations showed that the nuclear region of Arp 299-A hosts an extremely prolific supernova factory ([10] and Figure 1-left). We found that the radio luminosities of the compact objects in Arp 299-A were typical of normal RSNe, which result from the explosion of type IIP, IIb and IIL SNe. All these results confirmed that Arp 299-A was a very well suited target to eventually determine the complete CCSN rate in a local LIRG.

\subsection{Cohabitation of starburst and AGN in Arp 299-A, a microquasar candidate, and evidence for foreground (H II) absorbers}

The dusty nuclear regions of luminous infra-red galaxies are heated by either an intense burst of massive star formation, or an active galactic nucleus (AGN), or a combination of both. Disentangling the contribution of each of those putative dust-heating agents is a challenging task, and direct imaging of the innermost few pc can only be accomplished at radio wavelengths, using very high-angular resolution observations. Hence, after the successful observations that allowed us to unveil the supernova factory in Arp 299-A, we proposed further EVN observations at two frequencies, in the hope of unveiling the nature of the already discovered objects. The 1.7 and $5.0 \mathrm{GHz}$ contemporaneous EVN observations on Arp 299-A showed yet another remarkable result: the discovery of the long-sought AGN in Arp 299-A ([12] and Fig. 1-right). A1, the brightest compact source in Arp 299-A in our 5.0 GHz EVN observations, was also the brightest source at $1.7 \mathrm{GHz}$. The $1.7 \mathrm{GHz}$ EVN image showed also diffuse, low-surface brightness emission from A1 towards A5 (Figure 1-right). The morphology, radio luminosity, spectral index and ratio of radio-to-X-ray emission of the A1-A5 region is in perfect agreement with expectations from a low-luminous AGN (LLAGN). Since Arp 299-A had long been thought of as a pure starburst, our finding of a buried, LLAGN in its central region and cohabitating with a recent burst of star-formation, suggests that starburst and AGN phenomena may be frequently associated in mergers. 

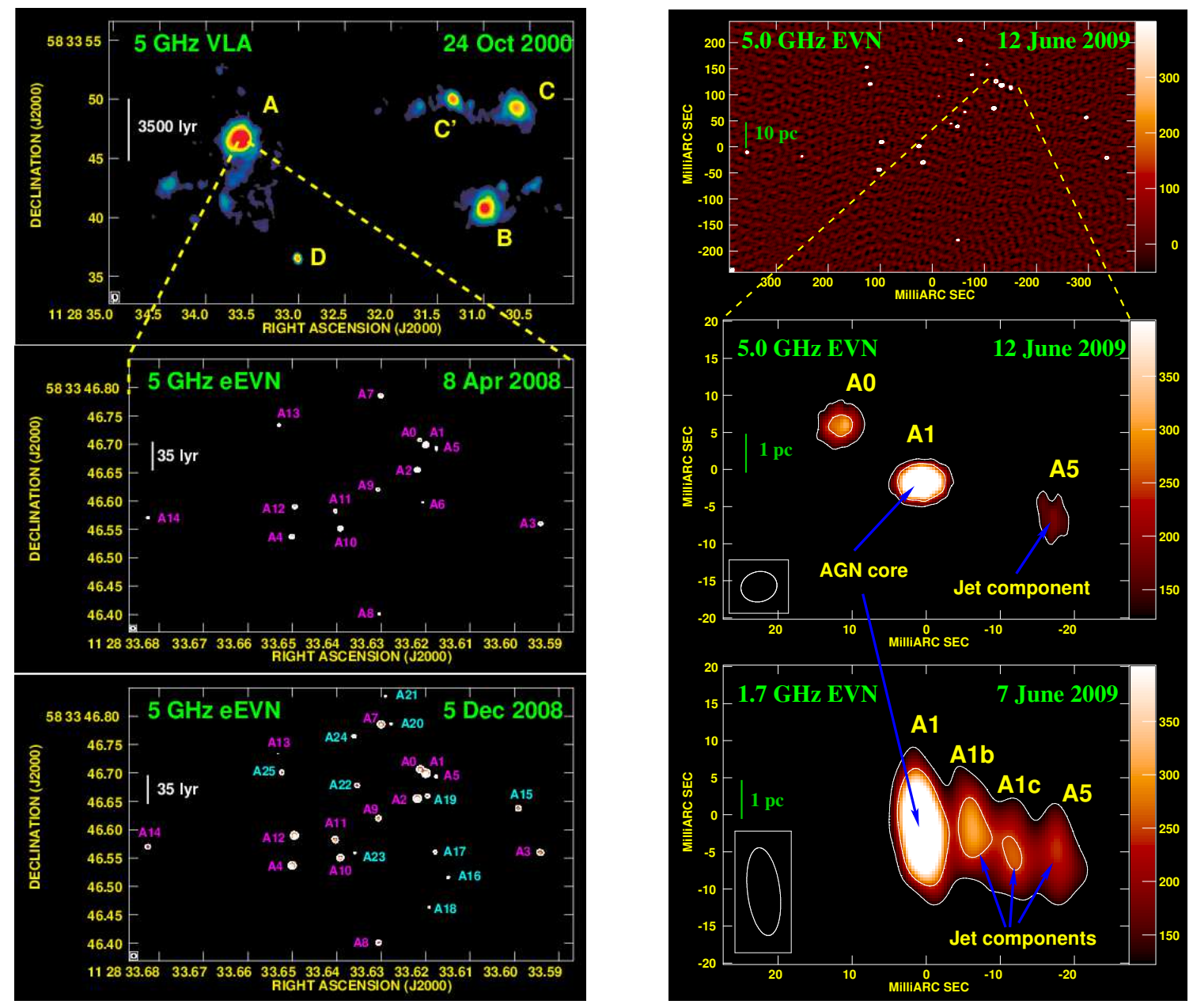

Figure 1: Top left: $5 \mathrm{GHz}$ VLA archival observations of Arp 299 on 24 October 2000, displaying the five brightest knots of radio emission in this merging galaxy. Middle and bottom left: Contour maps drawn at five times the r.m.s. noise of our $5 \mathrm{GHz}$ eEVN observations of the central 500 light years of the luminous infrared galaxy Arp 299-A on 8 April 2008 and 5 December 2008, revealing a large population of relatively bright, compact, non-thermal emitting sources. To guide the reader's eye, we show in cyan the components detected only at the 5 December 2008 epoch. Top right: $5.0 \mathrm{GHz}$ full EVN image of the central 150 parsec region of the luminous infrared galaxy Arp 299-A, displaying a large number of bright, compact, non-thermal emitting sources, mostly identified with young RSNe and SNRs. Middle and bottom right: Blow-ups of the inner 8 parsec of the nuclear region of Arp 299-A, as imaged with the full EVN at 1.7 and 5.0 GHz. The morphology, spectral index and luminosity of the A1-A5 region are clear evidence of a core-jet structure, typical of AGNs. Contours are drawn at 5 and 10 times the off-source r.m.s. noise. 
There is also growing evidence that A6 is a microquasar, based on its appearance and disappearance in our $5.0 \mathrm{GHz}$ images [12, 2]. If confirmed, A6 would be, together with the microquasar candidates in Arp 220 [1], one of the first extragalactic microquasars to be confirmed ever.

It appears that foreground absorbers, i.e., H II regions, are responsible for the low-frequency absorption of the synchrotron radio emission at frequencies around and below $1.7 \mathrm{GHz}$, for a significant number of sources. The most remarkable one is likely component A0, previously identified as a young RSN by [8], which is detectable at $5.0 \mathrm{GHz}$ more than $8 \mathrm{yr}$ after its discovery, but is undetected at $1.7 \mathrm{GHz}$ [12]. It is also remarkable that this RSN exploded at a mere (deprojected) distance of two parsecs from the putative AGN in Arp 299-A, which makes this supernova one of the closest to a central supermassive black hole ever detected. This result may also be relevant to accreting models in the central regions of galaxies, since it is not easy to explain the existence of very massive, supernova progenitor stars so close to an AGN. While seemingly contradictory, this could explain the low-luminosity of the AGN we see in Arp 299-A. In fact, since massive stars shed large amounts of mechanical energy into their surrounding medium, thereby significantly increasing its temperature, those massive stars would hinder the accretion of material to the central black hole, which could in turn result in a less powerful AGN than usual.

\subsection{The nature of the compact sources in Arp 299-A and their radial distribution}

More recently, we presented the first $5.0 \mathrm{GHz}$ radio light-curve (spanning $\sim 2.5 \mathrm{yr}$ ) of 26 compact components in the nuclear starburst of Arp 299-A ([2] and Fig. 1). The properties of all detected objects are consistent with them being a mixed population of CCSNe and SNRs. We obtained for the first time a direct estimate of CCSN rate in Arp 299-A of $\gtrsim 0.80 \mathrm{SN} / \mathrm{yr}$, indicating that the bulk of the current star formation in Arp 299-A is taking place in the innermost $\sim 150 \mathrm{pc}$. We have also used the VLBI data on Arp 299-A, together with that on Arp 220 and M 82 to probe for the first time the radial distribution of supernovae in the central regions of starburst galaxies. We have found that the surface density of SNe follows a power-law with radius, $\Sigma_{\mathrm{sn}} \propto r^{-1.0}$ [5]. This result gives support to theoretical work that predicts the formation of nuclear disks around supermassive black holes, and has important implications for understanding the co-evolution of AGN and starbursts in LIRGs.

\section{Summary}

Here, I have presented some of the results obtained by my team on the interacting system Arp 299, making use of the EVN, including the impressive discovery of an extremely prolific supernova factory in the central $\sim 150$ pc of the galaxy Arp 299-A ( $D=45 \mathrm{Mpc}$ ) and the monitoring of a large number of very compact radio sources in it, or the detection and precise location of the long-sought AGN in Arp 299-A. In addition, VLBI observations of distant U/LIRGs in the local universe have allowed us to estimate the energetics in magnetic fields and particles. We also used VLBI observations of the central $\sim 100$ pc of Arp 220 and Arp 299-A to show evidence for the existence of nuclear disks in them, which is a relevant result for models of co-evolution of AGN and starbursts in U/LIRGs.

Recently, we have detected the radio emission of SN 2010P [4] in Arp 299-C' (see Fig. 1-left). This SN was discovered at IR wavelengths, and it took more than a year to show up bright in 
radio. In Arp 299-B, we are monitoring the evolution of a bright radio "burp", whose nature is still unclear. All those results show that very-high angular resolution studies of nearby U/LIRGs are of high relevance for the comprehension of both local and high-z starforming galaxies, and make of Arp 299 an extraordinary laboratory in our local universe. No doubt, The Arp 299 show must go on!

\section{Acknowledgments}

I am grateful to the other members of the Arp 299 team (Antxon Alberdi, Marco Bondi, RubÃl'n Herrero-Illana, and Cristina Romero-Cañizales) for their help during different stages of this project. I warmly acknowledge financial support from the organizers of the 11th EVN Symposium through the RadioNet3 programme. This research has benefitted from financial support by the Spanish Government (MICINN and MINECO) through grants AYA 2009-13036-C02-01 and AYA 2012-38491- C02-02, cofunded with FEDER funds, as well as support by the Andalusian Autonomic Government through grants P08-TIC-4075 and TIC126. The European VLBI Network is a joint facility of European, Chinese, South African and other radio astronomy institutes funded by their national research councils.

\section{References}

[1] Batejat, F., Conway, J. E., Hurley, R., Parra, R., Diamond, P. J., Lonsdale, C. J., \& Lonsdale, C. J., Resolution of the Compact Radio Continuum Sources in Arp220, ApJ 740 (2011) 95

[2] Bondi, M., Pérez-Torres, M. A., Herrero-Illana, R., \& Alberdi, A., The nuclear starburst in Arp 299-A: from the 5.0 GHz VLBI radio light-curves to its core-collapse supernova rate, A\&A 539 (2012) A134

[3] Chevalier, R. A., The radio and X-ray emission from type II supernovae, ApJ 259 (1982) 302

[4] Herrero-Illana, R., Romero-Canizales, C., Pérez-Torres, M. A., Alberdi, A., Kankare, E., Mattila, S., \& Ryder, S. D., Radio detection of the near-IR discovered supernova 2010P, The Astronomer's Telegram 4432 (2012) 1

[5] Herrero-Illana, R., Pérez-Torres, M. Á., \& Alberdi, A., Evidence of nuclear disks in starburst galaxies from their radial distribution of supernovae, A\&A $\mathbf{5 4 0}$ (2012) L5

[6] Kennicutt, R. C., Jr., Star Formation in Galaxies Along the Hubble Sequence, ARA\&A 36 (1998) 189

[7] Magnelli, B., Elbaz, D., Chary, R. R., Dickinson, M., Le Borgne, D., Frayer, D. T., \& Willmer, C. N. A., Evolution of the dusty infrared luminosity function from $z=0$ to $z=2.3$ using observations from Spitzer, A\&A $\mathbf{5 2 8}$ (2011) A35

[8] Neff, S. G., Ulvestad, J. S., \& Teng, S. H., A Supernova Factory in the Merger System Arp 299, ApJ 611 (2004) 186

[9] Parra, R., Conway, J. E., Diamond, P. J., Thrall, H., Lonsdale, C. J., Lonsdale, C. J., \& Smith, H. E., The Radio Spectra of the Compact Sources in Arp 220: A Mixed Population of Supernovae and Supernova Remnants, ApJ 659 (2007) 314

[10] Pérez-Torres, M. A., Romero-Cañizales, C., Alberdi, A., \& Polatidis, A., An extremely prolific supernova factory in the buried nucleus of the starburst galaxy IC 694, A\&A 507 (2009) L17

[11] Pérez-Torres, M. A., Alberdi, A., Colina, L., Torrelles, J. M., Panagia, N., Wilson, A., Kankare, E., \& Mattila, S., Radio monitoring of NGC 7469: late-time radio evolution of SN 2000ft and the circumnuclear starburst in NGC 7469, MNRAS 399 (2009) 1641 
[12] Pérez-Torres, M. A., Alberdi, A., Romero-Cañizales, C., \& Bondi, M., Serendipitous discovery of the long-sought active galactic nucleus in Arp 299-A, A\&A 519 (2010) L5

[13] Romero-Cañizales, C., Mattila, S., Alberdi, A., Pérez-Torres, M. A., Kankare, E., \& Ryder, S. D., The core-collapse supernova rate in Arp 299 revisited, MNRAS 415 (2011) 2688

[14] Romero-Cañizales, C., Pérez-Torres, M. A., et al., e-MERLIN and VLBI observations of the luminous infrared galaxy IC 883: a nuclear starburst and an AGN candidate revealed, A\&A 543 (2012) A72 